\title{
Synthesis, characterization and in vivo evaluation of biocompatible ferrogels
}

\author{
M.T. Lopez-Lopez ${ }^{1,2^{*}}$, I.A. Rodríguez ${ }^{2,3}$, L. Rodriguez-Arco ${ }^{1,2}$, V. Carriel ${ }^{2,3}$, A.B. Bonhome- \\ Espinosa $^{1,2}$, F. Campos ${ }^{2,3}$, A. Zubarev ${ }^{4}$, J.D.G. Duran ${ }^{1,2}$ \\ ${ }^{1}$ Department of Applied Physics, University of Granada, Granada, Spain \\ ${ }^{2}$ Instituto de Investigación Biosanitaria ibs.GRANADA, Granada, Spain \\ ${ }^{3}$ Department of Histology (Tissue Engineering Group), University of Granada, Granada, Spain \\ ${ }^{4}$ Department of Mathematical Physics, Ural Federal University, Ekaterinburg, Russia
}

Keywords: Ferrogel; Polymer; Magnetic particle; Rheology; Magnetorheology; In vivo

\begin{abstract}
A hydrogel is a 3-D network of polymer chains in which water is the dispersion medium. Hydrogels have found extensive applications in the biomedical field due to their resemblance to living tissues. Furthermore, hydrogels can be endowed with exceptional properties by addition of synthetic materials. For example, magnetic field-sensitive gels, called ferrogels, are obtained by embedding magnetic particles in the polymer network. Novel living tissues with unique magnetic field-sensitive properties were recently prepared by 3-D cell culture in biocompatible ferrogels. This talk critically reviews the most recent progress and perspectives in their synthesis, characterization and biocompatibility evaluation. Optimization of ferrogels for this novel application requires low-density, strongly magnetic, multi-domain particles. Interestingly, the rheological properties of the resulting ferrogels in the absence of field were largely enhanced with respect to nonmagnetic tissues, which can only be explained by the additional cross-linking imparted by the embedded magnetic particles. Remarkably, rheological measurements under an applied magnetic field demonstrated that magnetic tissues presented reversibly tunable mechanical properties, which constitutes a unique advantage with respect to nonmagnetic tissues. In vivo evaluation of ferrogels showed good biocompatibility, with only some local inflammatory response, and no particle migration or damage to distant organs.
\end{abstract}

\section{Introduction}

Hydrogels are 3-D networks of hydrophilic polymer chains in which water is the dispersion medium. Hydrogels are called physical gels if the polymer network is maintained by physical interactions, whereas they are called chemical gels if chemical crosslinking has been created between polymer chains [1]. Depending on the nature of the interactions (either physical or chemical) that create and maintain the polymer network, hydrogels have different consistence - for example, physical gels often can be injected without breakage, whereas chemical gels cannot. However, no matter the kind of interaction behind the polymeric network, all hydrogels share some properties such as high water content, elevate porosity and soft consistency. These properties are very valuable from the point of view of biomedical applications, since they make hydrogels to resemble to living tissue. As a consequence, hydrogels are currently used for manufacturing contact lenses, tissue engineering scaffolds, drug delivery systems and wound dressings [1].

Hydrogels can be endowed with exceptional properties that do not possess living tissues by introducing synthetic materials. For example, hydrogels with ferromagnetic properties, called ferrogels, can be prepared by embedding magnetic particles in the polymer network [2-7]. Different approaches can be used for embedding magnetic particles in a polymer network. The simplest is soaking a hydrogel in a magnetic fluid [5]. However, in this case the magnetic particles are washed away when water is changed or when the hydrogel is plunged in water-based medium. Another possibility is to create the gel in the presence of magnetic particles and to ensure attraction between some groups of the polymer chain and proper functional groups on the surface of the particles, previously functionalized for this aim [2-4]. In such a way the particles remain trapped within the polymer network and stay even when the hydrogel is washed with water. Many advantages are reported for ferrogels in biomedical applications as compared with conventional (nonmagnetic)

\footnotetext{
*Corresponding author: modesto@ugr.es
} 
hydrogels. For example, ferrogels can be visualized and in-vivo followed-up by magnetic resonance imaging [8], and their magnetic moment under an applied magnetic field can be used to attract functionalized magnetic particles injected close to them in in-vivo applications $[5,8,9]$. Furthermore, when the embedded magnetic particles are micron-sized, the mechanical properties of the resulting magnetic hydrogels can be controlled by noncontact magnetic forces [10,11]. This smart property might be used, for example, to match the mechanical properties of the magnetic scaffolds to those of potential target tissues in tissue engineering applications [10].

In this paper we critically review our recent progress in the field of ferrogels as tissue-like models. First we will discuss on the protocol for the generation of the ferrogels. Then, we will present results on the rheological properties of the ferrogels. Finally we will analyze their biocompatibility in vivo.

\section{Preparation of magnetic field-sensitive hydrogels}

Magnetic hydrogels should at least contain magnetic particles, a polymer network and a water-based liquid as dispersion medium. As polymer material for the preparation of polymer network we used a mixture of human fibrin, resulting from the polymerization of human plasma (provided by the Granada Biobank of the Andalusian Regional Government, Spain), and type VII agarose (Sigma Aldrich, USA). This choice was motivated by its biocompatibility and proved use in tissue engineering applications [10]. The magnetic phase (magnetic particles) is the essential constituent that makes possible the control of the resulting hydrogel by magnetic forces. Two physical aspects of magnetic particles are key factors for this aim. Particles should be ferromagnetic with a strong enough saturation magnetization -magnetite is a good choice. Size of the particles is another key factor. Firstly, the size of the particles determines the clearance time and biodistribution of the particles in in vivo applications [12]. Second, particles of a size smaller than approximately $50 \mathrm{~nm}$ are too small so that magnetic interaction between them is always dominated by thermal motion, preventing from controllability of the mechanical properties by applied magnetic fields. On the contrary, as particle size increases the dispersion of the particles in the carrier is compromised due to the progressive loss of relevance of the thermal motion that gives rise to fast settling, avoiding obtaining homogeneous hydrogels. Based on the previous discussion we used commercial magnetite particles of an average diameter of $110 \mathrm{~nm}$ (MagP-OH, Nanomyp, Spain). These particles were provided with a biocompatible polymer coating, with $\mathrm{OH}^{-}$ functionalization, and have a saturation magnetization of $160 \mathrm{kA} / \mathrm{m}$ and negligible remnant magnetization [10]. Alternatively, we synthesized larger particles of $800-900 \mathrm{~nm}$ in diameter, a saturation magnetization of $150 \mathrm{kA} / \mathrm{m}$ and negligible remnant magnetization. In order to hinder phase separation by particle settling, we synthesized these magnetic particles by coating polymeric cores of an average diameter of $700 \mathrm{~nm}$ with a magnetite layer and an outer layer of PEG polymer [11] -we will refer to these particles as Poly@Mag@PEG. As we proved in a previous work, this configuration of nonmagnetic-core/magnetic-layer allows reducing mass density at the time that enhances the magnetic response at low and medium applied magnetic fields [13].

The magnetic hydrogels were generated by modification of a previously reported protocol for nonmagnetic hydrogels [14]. Briefly, for a final mixture volume of $5 \mathrm{~mL}$, we placed $3.8 \mathrm{~mL}$ of human plasma in culture disks and, afterwards, we added $625 \mu \mathrm{L}$ of a suspension of magnetic particles in PBS (with the adequate concentration of particles to fit the desired final concentration), $75 \mu \mathrm{L}$ of the solution of tranexamic acid, $0.25 \mathrm{~mL}$ of a mixture of agarose in PBS $(0.02 \mathrm{~g} / \mathrm{mL})$ and $250 \mu \mathrm{L}$ of the solution of calcium chloride. Afterwards, the mixtures were placed for about 10 hours in an incubator, at $37^{\circ} \mathrm{C}$ and with a flow of air enriched with $\mathrm{CO}_{2}$ at a concentration of $5 \%$. For the first 5 minutes of gelation we applied a vertical magnetic field of $36 \mathrm{kA} / \mathrm{m}$, with the help of a coil. Approximately $120 \mathrm{~min}$ after placing the samples in the incubator, we added $5 \mathrm{~mL}$ of PBS to the already jellified mixture. This bath of PBS prevented dehydration of the formed hydrogels caused by incubation temperature. For comparison we also prepared nonmagnetic hydrogels by following the same protocol without particle addition.

After preparation we extracted and characterized the jellified sample (approximately $1 / 3$ of the total volume). The magnetic gels were homogeneous in color and particles were not washed 
away even when the hydrogels were plunged in large amounts of water under the presence of a magnetic field. This proved that the particles were attached to polymer fibers.

\section{Physical characterization of the hydrogels}

We characterized the microstructure of the hydrogels by scanning electron microscopy (SEM). For the nonmagnetic hydrogel we observed an isotropic polymer network consisting of long fibrin fibers with some links (Figure 1a). In the case of the ferrogel prepared with MagP-OH particles (110 nm of mean diameter), we observed an anisotropic structure consisting of some thick braids of polymer fibers, approximately aligned in a given direction, separated by some regions with a lighter concentration of polymer fibers (Figure 1b). The presence of particles was not observed and, thus, we may induce that particles were embedded within the thick braids, which in fact should consist of a central core of particles and peripheral polymer fibers. Note that because of the magnetization of the particles, some alignment of the particle-fibrin composites might have took place during the first 5 min of gelling (magnetic field on), which can explain the observed alignment of the polymer braid. Finally, for ferrogels prepared with Poly@Mag@PEG particles (800-900 $\mathrm{nm}$ in diameter), we observed an isotropic structure of polymer fibers disrupted by the presence of the particles, homogeneously distributed within the polymer network (Figure 1c).
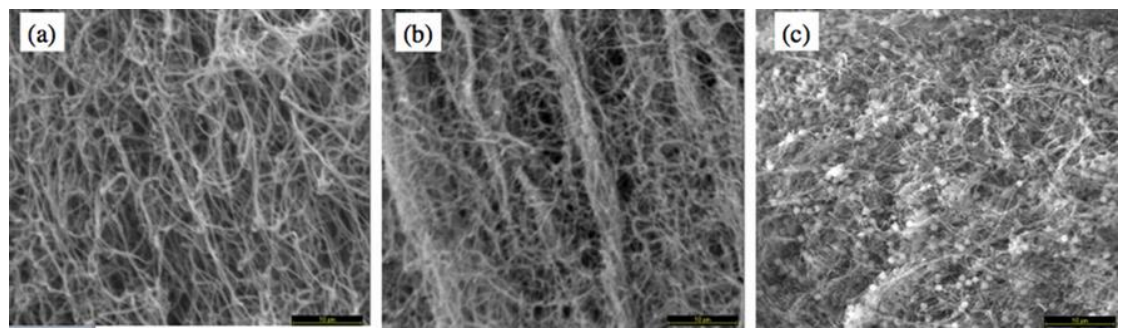

Figure 1. Scanning electron microscopy (SEM) picture of hydrogels. (a) nonmagnetic hydrogel;

(b) ferrogel prepared with MagP-OH particles; (c) ferrogel prepared with Poly@Mag@PEG particles. Bar length: 10 microns.

We characterized the magnetic properties of the ferrogels by VSM and SQUID magnetometry. The obtained magnetization curves (not shown here for brevity) showed soft ferromagnetic features, with values of saturation magnetization of approx. $2.67 \mathrm{kA} / \mathrm{m}$ and $1.5 \mathrm{kA} / \mathrm{m}$ for ferrogels prepared with MagP-OH particles and Poly@Mag@PEG particles, respectively. From these values of saturation magnetization we obtained the actual concentration of magnetic particles within the ferrogels on the basis of the mixing law [15], which turned out to be approx. 1.7 vol.\% for the ferrogel prepared with MagP-OH particles and 1.0 vol.\% for the ferrogel prepared with Poly@Mag@PEG particles.
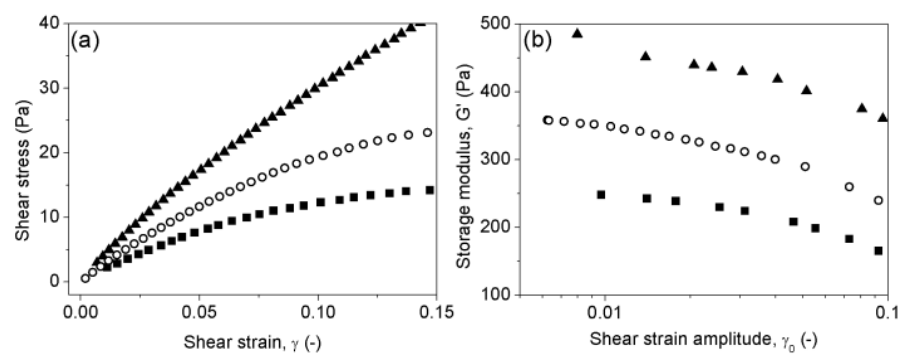

Figure 2. (a) Shear stress as a function of shear strain for hydrogels in stationary state. (b) Storage modulus as a function of shear strain amplitude for hydrogels under oscillatory shear of $1 \mathrm{~Hz}$ of frequency. Ш: Nonmagnetic hydrogel; O: ferrogel prepared with Poly@Mag@ PEG particles (1.0 vol.\% particle content); $\mathbf{\Delta}$ : ferrogel prepared with MagP-OH particles (1.7 vol.\% particle content).

Concerning the rheological properties, we characterized the steady state and dynamic behavior of hydrogels. For the steady state characterization we imposed a value of the shear strain and measured the corresponding value of the shear stress. Then, the value of the shear strain was increased stepwise and curves of the shear stress as a function of the imposed shear strain were 
obtained (Figure 2a). For the characterization of the dynamic regime we imposed oscillatory shear strain of fixed frequency $(1 \mathrm{~Hz})$ and increasing amplitude and measured the corresponding shear stress. From these measurements we obtained the values of the viscoelastic moduli as a function of the amplitude of the shear strain (Figure 2b). In all cases (results of G' not shown here for brevity), the storage modulus (G') was considerably higher than the loss modulus ( $G$ ' '), indicating that our hydrogels were more elastic than viscous, as expected for a cross-linked polymer systems.

In the stationary state, for a given value of the shear strain the corresponding shear stress was considerably higher for ferrogels than for the nonmagnetic hydrogel (Figure 2a). Results for the storage modulus ( $G^{\prime}$ ) show that this quantity was also considerably enhanced for ferrogels with respect to nonmagnetic hydrogels (Figure $2 b$ ) -note that similar results were obtained for G", not shown here. This enhancement of the mechanical properties of hydrogels when magnetic particles were included in the formulation correlated well with changes in the microstructure observed in Figure 1. In fact, this enhancement can only be explained by the additional cross-linking between polymer fibers imparted by the particles. We can suppose that particles attach strongly to different polymer fibers increasing the strength of the polymer network, in agreement with discussion of [16]. These authors reported that nanoparticle solutions can be used as adhesives for gels and biological tissues due to the nanoparticles' ability to adsorb onto polymer gels and to act as connectors between polymer chains. In fact, if particles are correctly functionalized, the linkage between particles and polymer is much stronger than the hydrogel itself. This is due to the ability of polymer chains to reorganize and dissipate energy under stress when adsorbed onto nanoparticles [16]. Concerning differences between values for ferrogels prepared with Poly@Mag@PEG particles and ferrogels prepared with $\mathrm{MagP}-\mathrm{OH}$, the latter presenting larger values of all rheological parameters, it should be noticed that the concentration of particles in MagP-OH ferrogels was higher (1.7 vol.\%) than in Poly@Pag@PEG ferrogels (1.0 vol.\%). In addition, other factors could play a role, like the different functionalization of particle surface or the particle size. A comprehensive analysis of the role of these factors will be the subject of future works.
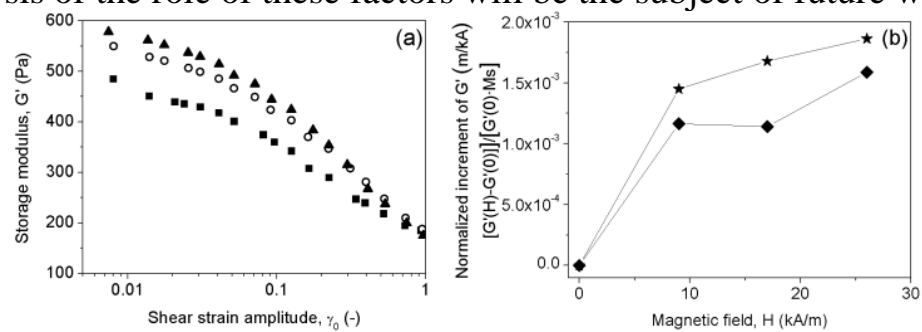

Figure 3. (a) Storage modulus as a function of the shear strain amplitude for hydrogel prepared with MagP-OH nanoparticles (1.7 vol.\%) under oscillatory shear of $1 \mathrm{~Hz}$ of frequency under the application of a magnetic field, $\mathrm{H} . \quad \mathbf{\square}: \mathrm{H}=0 \mathrm{kA} / \mathrm{m} ; \mathrm{O}: \mathrm{H}=9 \mathrm{kA} / \mathrm{m} ; \boldsymbol{\Delta}: \mathrm{H}=26 \mathrm{kA} / \mathrm{m}$. Adapted from [10]. (b) Normalization of the relative increment of $G^{\prime}$ with the field, [G'(H)- $\left.G^{\prime}(0)\right] / G^{\prime}(0)$, by the saturation magnetization of the particles, Ms, for ferrogels prepared with MagP-OH particles

$(\diamond)$ and ferrogels prepared with Poly@Mag@PEG particles (*). Adapted from [11].

Finally, we investigated the rheological response of our ferrogels under the application of a magnetic field. As observed, the storage modulus of ferrogels prepared with MagP-OH nanoparticles increased with the intensity of the magnetic field (Figure 3a). Similar results were obtained for other rheological parameters and for ferrogels prepared with Poly@Mag@PEG particles, not shown here for brevity. Thus, we can conclude that our ferrogels showed a typical magnetorheological (MR) effect. As discussed in Ref. [13], for a same amount of magnetic material in dispersion, the magnetic response, and thus the MR effect, can be enhanced at low and medium applied field by distribution of the magnetic material around a nonmagnetic core. This can be checked for our ferrogels by plotting a normalization of the field-induced increase of G' by the magnetic content (Figure 3b). As observed, the ferrogels prepared with Poly@Mag@PEG nanoparticles presented higher values of this normalization, from which we can conclude that the MR effect is comparatively stronger for these ferrogels. 


\section{In vivo evaluation of the ferrogels}

In vitro analysis with human fibroblast demonstrated that the presence of $\mathrm{MagP}-\mathrm{OH}$ or Poly@Mag@PEG nanoparticles did not affect cell viability and proliferation in ferrogels [10,11]. In addition, we performed in vivo analyses with animal models (mice and rats) to evaluate the biocompatibility of the ferrogels. We surgically implanted ferrogels in the subcutaneous connective tissue of the interscapular region of mice and rats. After surgery, all the animals were housed in a temperature-controlled room $\left(21 \pm 1{ }^{\circ} \mathrm{C}\right)$, provided with a $12 \mathrm{~h} \mathrm{light/dark} \mathrm{cycle} \mathrm{and} \mathrm{ad} \mathrm{libitum}$ access to tap water and standard mice/rat chow. Animals were maintained under these conditions for up to 5 weeks and were euthanized afterwards. Control animals (without ferrogel implantation) were also monitored for comparison. During the whole duration of the experiments, animals did not show any signs of side effects and the changes of body weight were similar to those of the control animals. The host response to the ferrogel implantation was a moderate acute local inflammatory reaction that progressively disappeared after the first week of implantation. Potential migration of particles to distant organs (kidney, liver, spleen, lung) was investigated before euthanasia by magnetic resonance imaging and after by VSM of organs' tissues, without appreciable migration within the limit of sensibility.

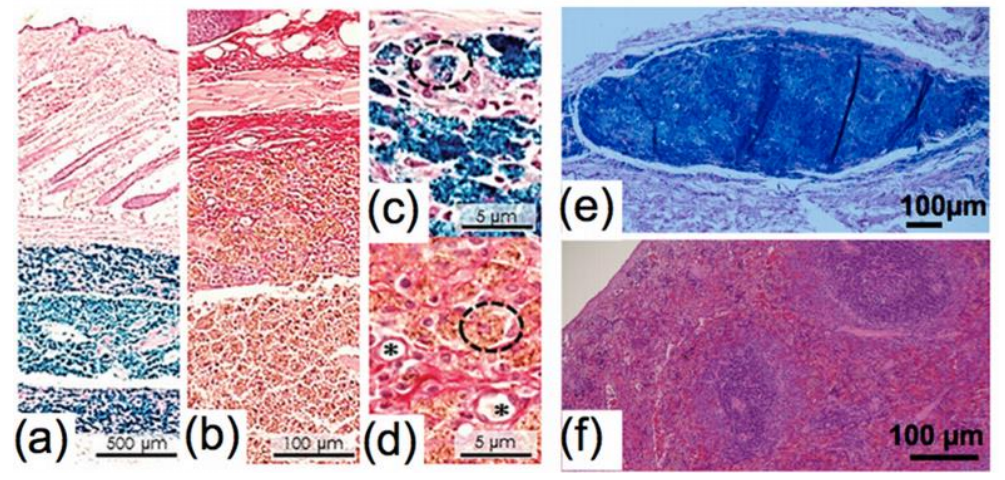

Figure 4. In vivo histological analyses. (a-d) Results for ferrogels prepared with Poly@Mag@PEG nanoparticles after 3 weeks of implantation in the connective tissue of the interscapular region of mince. (e-f) Results for ferrogels prepared with MagP-OH nanoparticles after 5 weeks of implantation in the subcutaneous tissue of the forelegs of rats. (a), (c) and (e) images show the Prussian blue histochemical reaction for the nanoparticles (Perl's method). (d) Show picrosirius staining. Note the presence of cells in the implanted ferrogels. Circles indicate macrophages, and (*) show small blood vessels. (f) Histological analysis of spleen shows normal pattern -all organs were histological normal. (a-d) were adapted from [11].

Histological analyses showed that host cells were able to invade the implanted constructs (Figure 4). In addition, as observed, the particles appeared homogeneously distributed over the ferrogels after several weeks of implantation (Figure 4). Histological analysis also confirmed that distant organs presented normal pattern, without appreciable damage for the duration of the experiments (Figure 4f).

\section{Conclusions}

We have reported the preparation of two kinds of water-based ferrogels consisting of magnetic nanoparticles of two types embedded within a fibrin polymer network. Scanning electron microscopy revealed that the presence of particles modified the microstructure of the ferrogels with respect to nonmagnetic hydrogels. Changes in the internal microstructure correlated with differences in the rheological properties. In particular, ferrogels presented superior mechanical properties as compared with nonmagnetic hydrogels -all rheological parameters were considerably enhanced with respect to nonmagnetic hydrogels, very likely as a consequence of additional crosslinking provided by the magnetic nanoparticles. What is more, the rheological properties of our ferrogels showed reversible controllability by noncontact magnetic forces. Finally, we evaluated the biocompatibility of the ferrogels in vivo by chirurgical implantation in mince and rats. Interestingly, the implantation only resulted in a localized and transitory acute inflammatory 
reaction, without affecting distal organs, which demonstrates the potential of ferrogels as tissue-like models for in vivo applications.

\section{Ethics statement}

This study was approved by the Ethics Committee of the University of Granada, Granada, Spain. The in vivo characterization was performed following the European Union and Spanish Government guidelines for ethical care of animals (EU Directive no. 63/2010, RD 53/2013).

\section{Acknowledgments}

Financial support: projects FIS2013-41821-R and FIS PI14-1343 (MINECO, Spain; co-funded by ERDF, UE). AZ also acknowledges the Russian Scientific Foundation, project14-19-00989.

\section{References}

[1] E. Caló, V.V. Khutoryanskiy. European Polymer Journal, 65 (2015) 252.

[2] R. Messing, N. Frickel, L. Belkoura, R. Strey, H. Rahn, S. Odenbach, A.M. Schmidt. Macromolecules 44, 2990-2999 (2011).

[3] P. Ilg. Soft Matter 9, 3465-3468 (2013).

[4] S.V. Berkum. PhD Thesis, Promotor: AP Philipse. Universiteit Utrecht (2014).

[5] N. Bock, A. Riminucci, C. Dionigi, A. Russo, A. Tampieri, E. Landi, V.A. Goranov, M. Marcacci, V. Dediu. Acta Biomaterialia 6, 786-796 (2010).

[6] O. Ozay, S. Ekicia, Y. Barana, N. Aktasb, N. Sahinera. Water Research 43, 4403-4411 (2009).

[7] J. Qin, I. Asempah, S. Laurent, A. Fornara, R.N. Muller, M. Muhammed. Advanced Materials 21, 1354-1357 (2009)

[8] O. Ziv-Polat, H. Skaat, A. Shahar, S. Margel. International Journal of Nanomedicine 7, 1259-1274 (2012).

[9] S. Panseri, C. Cunha, R. D'Alessandro, M. Sandri, G. Giavaresi, M. Marcacci. Journal of Nanobiotechnology 10, 32 (2012).

[10] M.T. Lopez-Lopez, G. Scionti, A.C. Oliveira, J.D.G. Duran, A. Campos and M. Alaminos, PLoS ONE 10, e0133878 (2015).

[11] L. Rodriguez-Arco, I.A. Rodriguez, V. Carriel, A.B. Bonhome-Espinosa, F. Campos, P. Kuzhir, J.D.G. Duran, M.T. Lopez-Lopez. Nanoscale 8, 8138-8150 (2016).

[12] F. Alexis, E. Pridgen, L.K. Molnar, O.C. Farokhzad. Molecular Pharmaceutics 5, 505-515 (2008).

[13] L. Rodriguez-Arco, M.T. Lopez-Lopez, P. Kuzhir, G. Bossis, J.D.G. Duran, ACS Applied Materials \& Interfaces, 5, 12143-12147 (2013).

[14] M. Alaminos, M.C. Sanchez-Quevedo, J.I. Muñoz-Avila, D. Serrano, S. Medialdea, I. Carreras, A. Campos. Investigative Ophthalmology \& Visual Science 47, 3311-3317 (2006).

[15] R.E. Rosensweig. Ferrohydrodynamics. Cambridge: Cambridge University Press; 1985.

[16] S. Rose, A. Prevoteau, P. Elziere, D. Hourder, A. Marcellan, L. Leibler. Nature 505, 382385 (2014). 